\title{
Initial Performance of the SLD Cherenkov Ring Imaging Detector System ${ }^{i}$
}

\author{
K. Abe, ${ }^{a}$ P. Antilogus, ${ }^{b, i i}$ D. Aston, ${ }^{b}$ K. Baird, ${ }^{c}$ A. Bean, ${ }^{d}$ T. Bienz, ${ }^{b, i i i}$ F. Bird, ${ }^{b, i v}$ D. O. Caldwell, ${ }^{d}$ \\ M. Cavalli-Sforza, ${ }^{e}$ P. Coyle, ${ }^{e, v}$ D. Coyne, ${ }^{e}$ S. Dasu, ${ }^{b, v i}$ S. Dolinsky, ${ }^{b, v i i}$ A. d'Oliveira, ${ }^{f, v i i i}$
} J. Duboscq, ${ }^{d}$ WW. Dunwoodie, ${ }^{b}$ P. Gagnon, ${ }^{e}$ G. Hallewell,,${ }^{b}$ K. Hasegawa, ${ }^{a}$ J. Huber, ${ }^{d}$ P. Jacques ${ }^{c}$ R. A. Johinson, ${ }^{f}$ M. Kalelkar, ${ }^{c}$ H. Kawahara,${ }^{b}$ Y. Kwon,${ }^{b}$ D. W. G. S. Leith,${ }^{b}$ X. Liu, ${ }^{e}$ A. Lu, ${ }^{d}$ J. Martinez, ${ }^{f}$ 'L. Mathys, ${ }^{d}$ S. McHugh, ${ }^{d}$ B. Meadows, ${ }^{f}$ D. Muller,${ }^{b}$ T. Nagamine,${ }^{b}$ M. Nussbaum, ${ }^{f}$ T. J. Pavel, ${ }^{b}$ R. Plano, ${ }^{c}$ B. Ratcliff, ${ }^{b}$ P. Rensing, ${ }^{b}$ A. K. S. Santha, ${ }^{f}$ D. Schultz, ${ }^{b}$ J. Shank, ${ }^{g}$ S. Shapiro, ${ }^{b}$ A. Shoup ${ }^{f, i x}$ C. Simopoulos, ${ }^{b}$ M. Sokoloff, ${ }^{f}$ E. Solodov, ${ }^{b, v i i}$ P. Stamer, ${ }^{c}$ I. Stockdale,${ }^{f, x}$ F. Suekane,${ }^{a}$ N. Toge,${ }^{b}$ J. Va'vra,${ }^{b}$ J. S. Whitaker,${ }^{g}$ D. A. Williams, ${ }^{e}$ S. H. Williams, ${ }^{b}$ M. Witherell, ${ }^{d}$ R. J. Wilson, ${ }^{g}$ G. Word, ${ }^{c}$ S. Yellin, ${ }^{d}$ and H. Yuta ${ }^{a}$

\author{
aDepartment of Physics, Tohoku University, Aramaki, Sendai 980, JAPAN \\ ${ }^{b}$ Stanford Linear Accelerator Center, Stanford University, Stanford, CA 94309, USA \\ 'Serin Physics Laboratory, Rutgers University, P.O. Box 849, Piscataway, NJ 08855, USA \\ ${ }^{d}$ Department of Physics, University of California, Santa Barbara, CA 93106, USA \\ ${ }^{e}$ Santta Cruz Institute for Particle Physics, University of California, Santa Cruz, CA 95064, USA \\ ${ }^{f}$ Department of Physics, University of Cincinnati, Cincinnati, OH 45221, USA \\ ${ }^{\circ}$ Department of Physics, Boston University, Boston, MA 02215, USA
}

\section{Abstract}

All of the major subsystems for the barrel Cherenkov Ring Imaging Detector (CRID) in the SLD at SLAC have now been commissioned. The CRID participated in the SLD engineering run of June-August 1991. In a cosmic ray test at the end of the run, Cherenkov rings were observed for the first time. Initial data from the CRID, including Cherenkov rings, studies of minimum ionizing particles, and clata from the fiber optics calibration system are presented here.

\footnotetext{
'Work supported by Department of Energy concract DE-ACO3. 76 SF 00515 and National Science Foundation grants NoB. PHY8813669 and PHY88-13018.

iPresent Address: Inst. de Physique Nucleaire, $43 \mathrm{Bd}$. du 11 Novembre 1918, 69622 Villeurbanne, France.

iii Present Address: Dept. of Physics and Astronomy, University of Iowa, Iowa City, IA 52242, USA.

iv Present Address: SSC Laboratory, 2550 Beckleymeade Ave., Suite 125, Dallas, TX 75237, USA.

'Plresent Address: Centre de Physique des Particules, Faculte des Sciences de Luminy, 13288 Marseille, France.

vi Sper.ker.

vii Pprmanent Address: Inst. of Nuclear Physics, Novosibirsk, 630090, USSR.

viii Permanent Address: Universidade Estadual Paulista, UNESPLampus de Guaratingueta, 12500 Sao Paulo - SP Brazil.

'x Present Address: Dept. of Physics, University of Califomia, Irvine, CA 92717, USA.

${ }^{x}$ Present Address: NASA Ames Research Center, MS 258-6, Moffett Fieid, CA $94035-1000$, USA.
}

\section{INTRODUCTION}

The SLD barrel Cherenkov Ring Imaging Detector (CRID) system [?] is designed to allow excellent particle identification in the study of $Z^{0}$ physics at the SLAC Linear Collider (SLC). It uses a combination of liquid and gas fluorocarbon radiators to allow $\pi / \mathrm{K} / \mathrm{p}$ separation up to $30 \mathrm{GeV} / \mathrm{c}$, and e/ $\pi$ separation up to $6 \mathrm{GeV} / \mathrm{c}$. The Cherenkcv photons are focused onto quartz drift boxes, as shown in Figure 1, where they are converted to electrons by $0.1 \%$ concentration of tetrakis(dimethylamino)ethylene (TMAE) in ethane gas. The single photoelectrons are drifted by a graded electric potential to a multiwire proportional chamber. The hit wire number, drift time and charge division measurements on $7 \mu \mathrm{m}$ carbon fibers are used to reconstruct the Cherenkov rings.

\section{ENGINEERING RUN STATUS}

The CRID components and support systems [?] were commissioned and tested during the engineering run of JuneAugust 1991. All 400 mirrors [?], 40 liquid radiator trays, drift boxes and detectors were installed a year ago. The fluid delivery systems were commissioned along with their monitoring systems [?]. Front end electronics packages [?] were installed on 22 of the 40 detectors. Lack of access to the SLD when the run began, prevented the installation of 


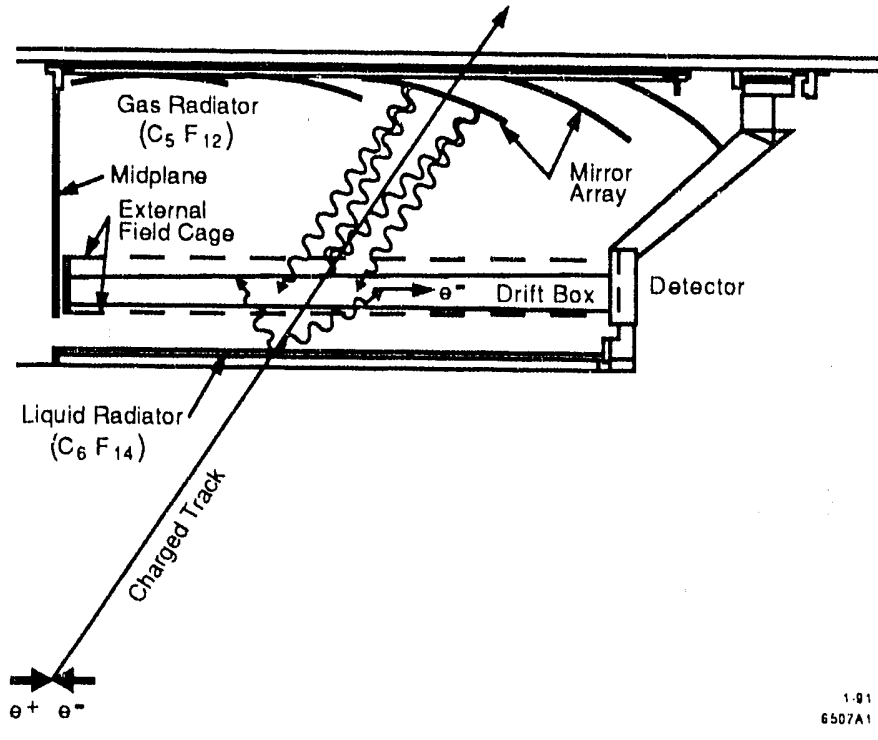

Figure 1: Quarter section of the SLD barrel CRID showing its principal components.

the rest of electronics packages. The front end electronics, FASTBUS data acquisition modules, and software systems were debugged during the rum. The fiber optics calibration system was in place and was used extensively to test the performance of the drift boxes. The temperature control and monitor system was also in place and was operated at $30^{\circ} \mathrm{C}$, instead of the nominal $40^{\circ} \mathrm{C}$, because thermal insulation was not completely installed to protect the SLIJ Irift chambers.

The vessel was filled with $\mathrm{CO}_{2}$ gas, in place of the $\mathrm{C}_{5} \mathrm{~F}_{12}$ gas radiator, to allow high voltage operation while the radiator fluid systems were being commissioned. All drift hoxes and detectors [6] were tested successfully at full voltage before the run began.

'The drift gas delivery system supplied CP-grade ethane after passing it through filters to remove oxygen, water vapor and other contaminants that reduce the electron lifetine or otherwise affect the CRID components. The quality of the gas was monitored on return lines from the drift. hoses by measuring electron lifetimes, UV transparency, and trace oxygen and water vapor content. The oxygen content in the drift gas return lines was measured before int roducing TMAE into the system. These data, shown in Figure 2(a), indicate that, for most of the drift boxes, oxygen level is aboul $0.7 \mathrm{ppm}$. However, there are six bexes whose oxygen levels are significantly larger than the average, indicating a small leak. That the effects were due to leaks in the return lines, was verified by varying the gas flow through those lines. It is of interest to note that the oxygen meter [7], when used to make a relative meastrement, can detect oxygen leaks at the 0.3 (0.5 pom level

The low oxygen and water vapor levels gave us enongh confidence to introduce TMAE into the drift boxes. The drift gas was doped with TMAE, by bubbling the gas

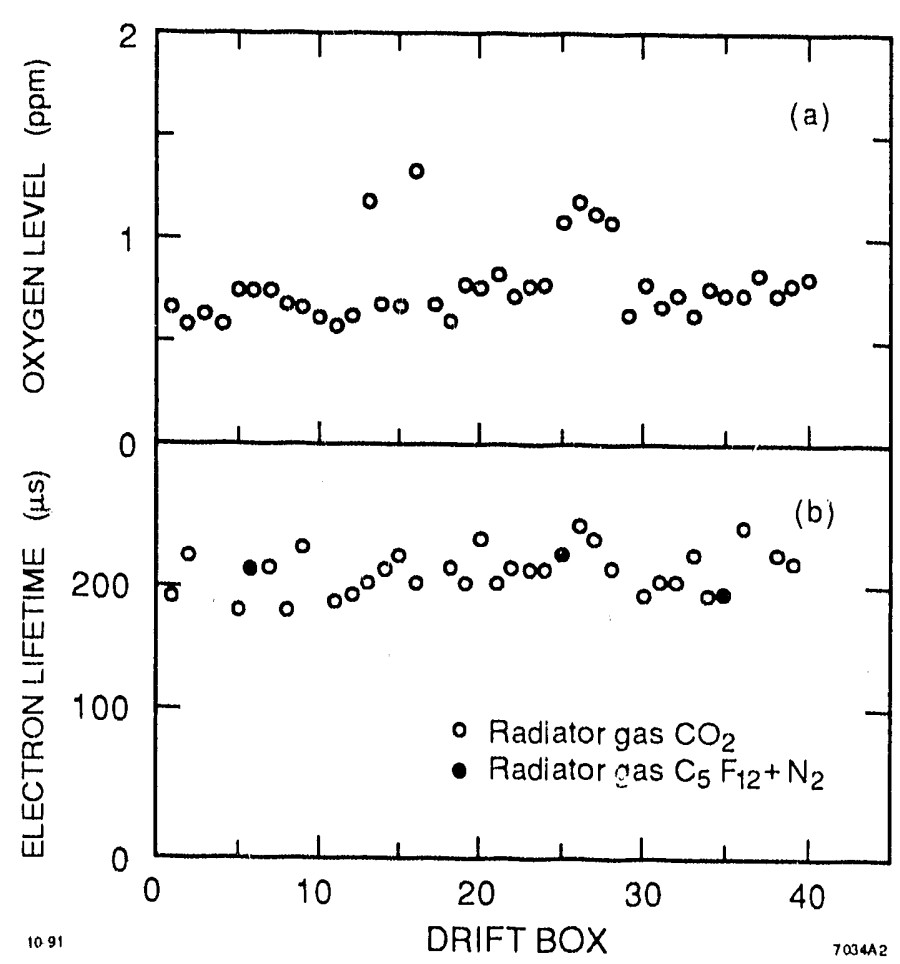

Figure 2: (a) 'Trace oxygen levels before the introduction of TMAE for all 40 drift box return lines. (b) Electron lifetimes as measured by a miniature ionization chamber for the return gas from all the drift boxes. The radiator gas was $\mathrm{CO}_{2}$ for the measurements shown as open circles. The lifetime did not change when highly electronegative $\mathrm{C}_{5} \mathrm{~F}_{12}$ was introduced into the CRID vessel, as indicated by the dark circles.

through $15^{\circ} \mathrm{C}$ liquid ' $\mathrm{TMAE}$, and was supplied to the drift boxes in the middle of the engineering run. The nominal TMAE temperature is $27^{\circ} \mathrm{C}$, but we chose to be conservative and run the system at lower temperatire. Electron lifetimes were measured using a miniature inn chanber [8]. These measurements, shown in Figure 2(b), are consistent with our previous test bench resulis for the cleanest drift gas doped with 'TMAF. The lifetime did not degrade even when highly electronegative $C_{5} F_{12}$ gas was introduced into the gaseous radiator volume in the later part of the run, as indicated by the dark points in Figure 2(b). Based on these measurements, it appears that the loaks detected by the oxygen meter are too small to affect the rectron lifetime.

By the end of the rum, the gas radiator recirculation system was commissioned, A 50 -50 mixtme of $\left(\mathrm{C}_{5} \mathrm{~F}_{12}\right.$ and $\mathrm{N}_{2}$ gases was introduced into the (:RID) vessel. 'This gas mixture lats a comdensation point below room temperature, and was, therefore, salfe to use while the (:RID) was mathtained at $30^{\circ} \mathrm{C}$. The homogenerty of the gas mixtmen was determined to be better than $1 \%$ by somar monitoring [4].

The liquid radiator recirculation system was also com. missioned at the end of the run. The quality of the liyuid was monitored extensively oflline hy measuring its If transparency. Six trays were then fillod with ( ${ }_{i} F_{1.4}$ liy- 


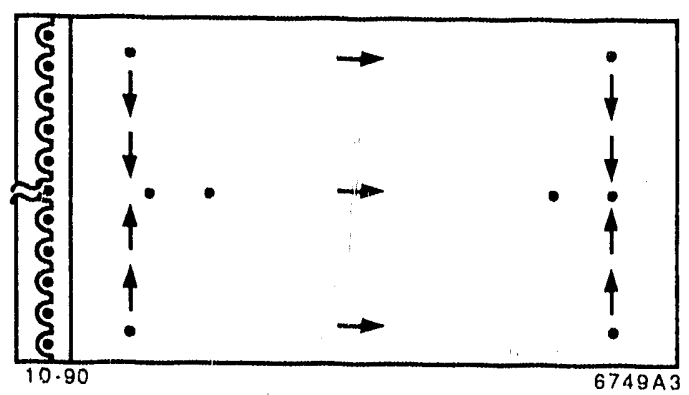

Figure 3: A schematic drawing of the placement of UV optical fibers on the drift boxes. The dots indicate fibers placed perpendicular to the quartz surface of the box. The arrows indicate the direction of the $45^{\circ}$ plane in which the beam from the fiber is aimed.

uid. However, only a fraction of these trays were situated in front of detectors with fully opcrating electronics.

Early in the run, before the radiators were commissioned, charged tracks produced in $e^{+}-e^{-}$collisions were observed clearly, by detecting the ionization left by them in the CRID. These data demonstrated that the electronics and the data analysis programs worked adequately [5]. However, we were unable to obtain Cherenkov ring data at that time. When the radiator fluids were available, dedicated CRID tests were conducted and their results are described below.

\section{UV FIBER RESULTS}

A UV fiber optic system [2] attached to all 40 drift boxes is used for the purpose of continuously monitoring drift velocities, measuring drift path distortions, and checking, the charge division on the anode wires. In this system, a UV flashlamp illuminates a set of optical fiber bundles which transmit the UV signal to the drift boxes. 'The arrangement of fibers on each drift box is shown in Figure 3 . 'There are three different orientations: eight beams are directed perpendicular to the quartz window (indicated by (lots in the figure) and are used to monitor the electron drift velocity. The remaining fibers are angled at $455^{\circ}$ with respect to the quartz window, in the planes indicated by the arrows. 'The four fibers closest to the detector are used to check the charge division calibration of the wires. The pemaining angled fibers are used to measure drift path distortions in the depth coordinate. All fibers are used to measure distortions in the width coordinate.

The single photoclectrons produced by hile light from the fibers are useful for a mumber of diagnostic and commissioning purposes. As soon as the TMAE (opant, was int roduced into the drift gas, the single electron hits were retected. 'The pattern of these hits, shown in Figure 4, is as expected. 'This fact demonstrates mot only that single acetrons are olserved, but also that the charge division conerlinate is being woll reconstructed, and that there are no surious distortions in the drift paths. The data from the fibers placed at various positions along the box are used to

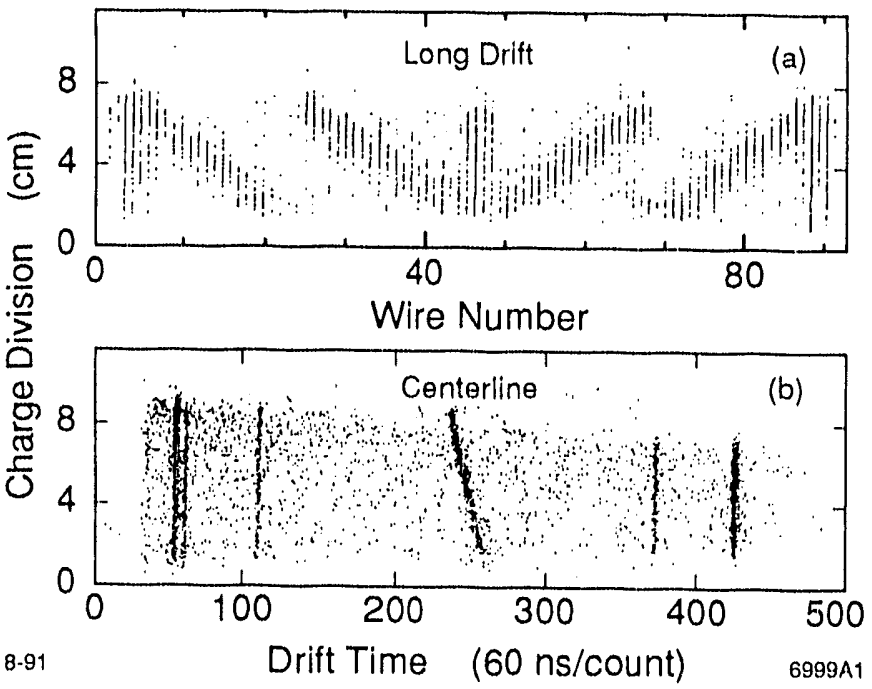

Figure 4: Representative results from UV fibers on drift boxes: (a) in a plane parallel to the detector wire plane; and (b) in a plane down the middle of the drift box.

measure the drift velocity. The drift velocity data shown in Figure 5 are better than the required $0.1 \%$ accuracy. These measurements were made with dedicated fiber runs immediately following the engincering run. Each point, is based on at most the amount of data expected in one hour of SLD data running and is the average over the 17 operational drift boxes. The error bars are statistical only. The expected slow variations in drift velocity will be tracked to better than the required accuracy.

\section{Cosmic Ray Test Results}

During the cosmic ray run, when gas and licquid radiators were in place, we observed candidate Clierenkov rings. The cosmic ray muon trigger was provided by the SLI) muon calorimeter, and the data were collected for the CRID and the central drift chamber. Many of the several hundred events collected were scanned individually to identily candidate (heronkov rings, and a sample sel, of events was determined. These events were reanalyzed oflline with full tracking and CRID analysis.

The liyud ring data sample is rather limited because? triggered cosmic rays passed through only one filled liquid radiator tray. The (IRID) offline analysis program extranolates the reconstructed drift chamber tractis to the middla of the liguid radiator tray. For each (JRII) hit, the program then computes the polar angles with respect to the drift chamber track direction, so that a Cherenkov photon emitted by the charged particle would make a hit at the observed point. 'These reconstructed cherentiov photont

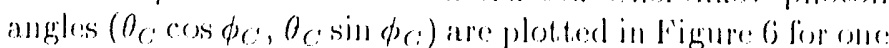
such event. 'The resolution of the angle Oe: was determined by using the complete data set to bo $\sim 25$ mad, and $2 \sigma$ contours of the expected Cherenkov angle are also plotted on Figure 6. Note that in this particulat cosmice ray event. 


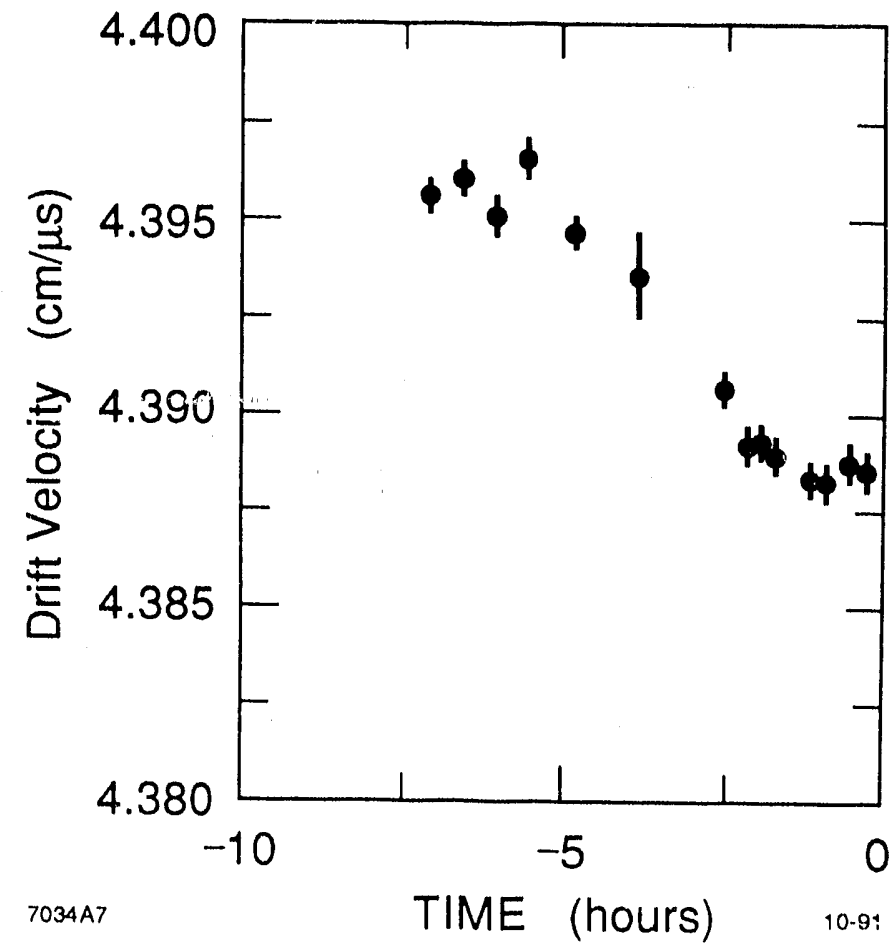

Figure 5: Measured drift velocity, averaged over 17 working drift boxes, as a function of time, demonstrating the ability to measure and calibrate this quantity with the fiber optic system. An overall systematic error of $\pm 0.002 \mathrm{~cm} / \mu \mathrm{s}$ is not shown.

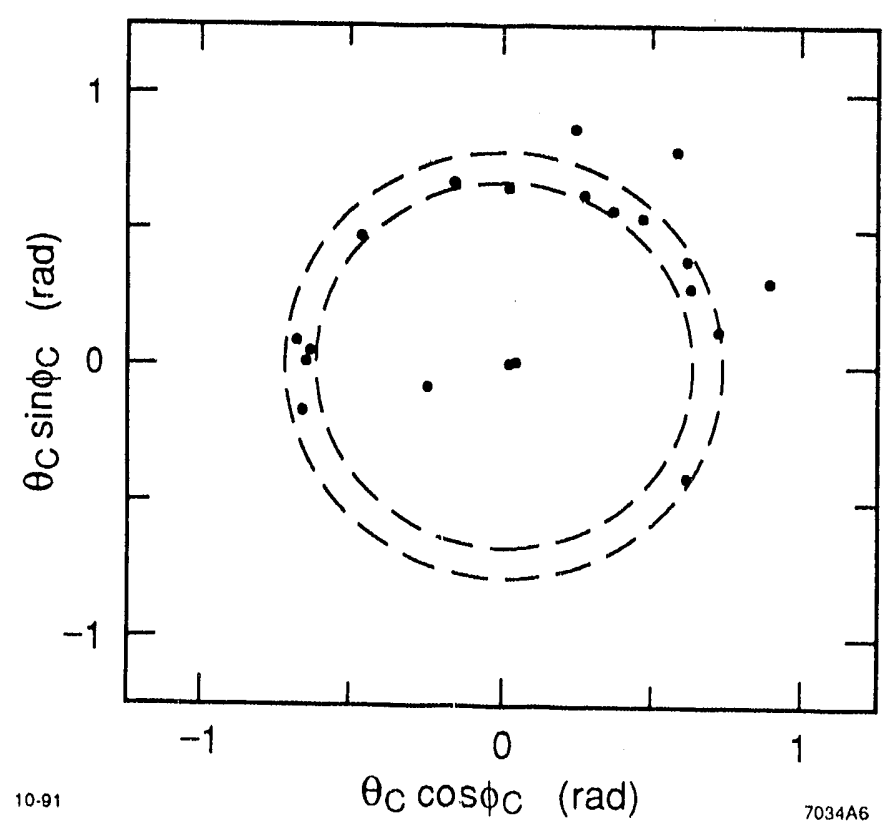

Figure 6: Reconstructed liquid Cherenkov angles for a cosmic ray event. The points near the origin are hits associated with the minimum ionization particle signal from the muon. The dotted lines represent the $\pm 2 \sigma$ expected region for Cherenkov photons. There is very little background. Two of the three hits in the upper right corner are consistent with radiation from the quartz window on the liquid radiator tray.

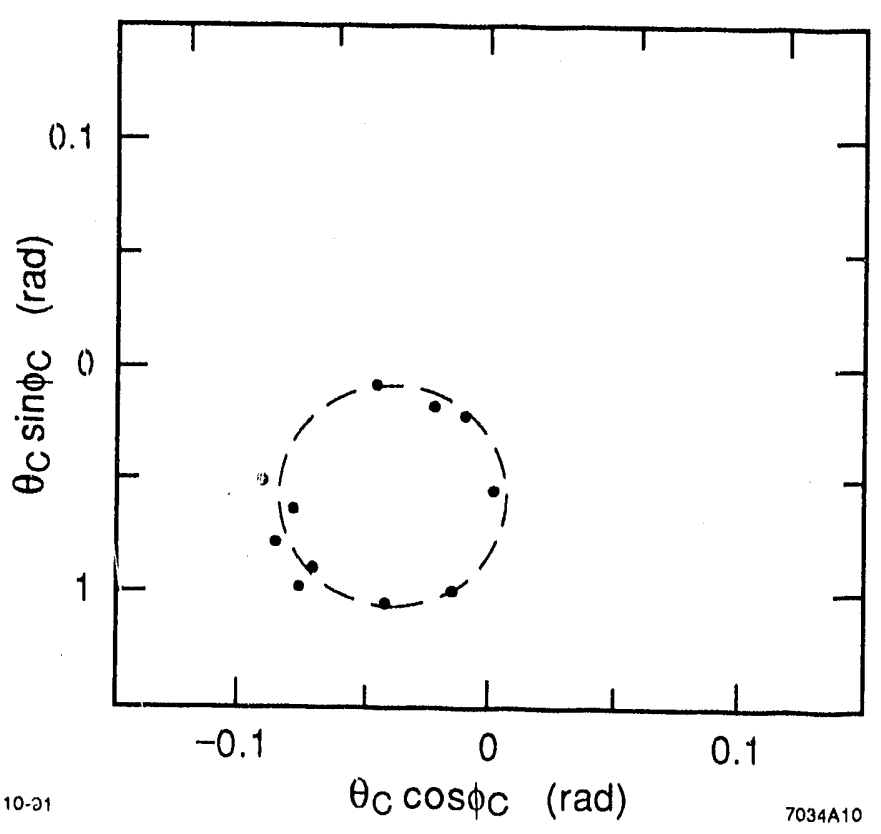

Figure 7: Reconstructed gas Cherenkov angles for a cosmic ray event along with a ring having the expected radius of 45 mrad. 'The mirror alignment is still under study and is expected to account for the shift in the ring from the origin.

the muon track crossed the liquid radiator tray perpendicular to the quartz window. The gap in the Cherenkov ring is due to the gap between the two drift boxes. In a typical $e^{+}-e^{-}$collision event, tracks originate from the interaction point, and cross the tray at an angle. For these more typical tracks, there is a cutoff in the $\phi_{C}$ angle due to total internal reflection.

We have also identified candidate gas rings from this data sample. The reconstructed Cherenkov angles from one such gas ring are plotted in Figure 7. The expected $\theta_{C}$ is $45 \mathrm{mrad}$. A circle of this radius centered in the middle of the hits is also shown in Figure 7. The mirror alignment is still under study and is expected to account for the shift in the ring from the origin. We are also studying the alignment of the CRID system with the central drift chamber using these cosmic ray data.

\section{Conclusion}

The SLD Engineering run and the ensuing dedicated CRID test runs have demonstrated that the system belaves as expected. We have been able to track particles using ionization in the drift boxes, and reconstruct. single electron coordinates very well. We have also reconstructed Cherenkov rings in the cosmic ray test. The runs have also enabled debugging of this complex system. We are now in the process of completing the installation of the remaining electronics packages, and refining the data accuisition and analysis software. We expect to have a complete (RID) for the next. SLD) (latia run. 


\section{REFERENCES}

[1] "SLD Design Report," SLAC-273, UC-43D (1984), and revisions.

[2] P. Antilogus et al., "Component and System Tests of the SLD Cherenkov Ring Imaging Detector," SLACPUB-5365, contributed to 1990 IEEE Nucl. Sci. Symp., Arlington, VA (1991).

[3] K. Abe et al., "Production of 400 Mirrors with High UV Reflectivity for use in the SLD Cherenkov Ring Imaging Detector," Nucl. Instr. and Meth. A300 (1991) 501.

[4] P. A.ntilogus et al., "Monitor and Control Systems for the SLD Cherenkov Ring Imaging Detector," Nucl. Inst.r. and Meth. A293 (1990) 136.
[5] P. Antilogus et al., "Cherenkov Ring Innaging Detector Front-End Electronics," IEEE Trans. Nucl. Sci. NS38 (1991) 408; and K. A be et al., "Performance of the Front-End Electronics and Data Acquisition for the SLD Cherenkov Ring Imaging Detector," contribution to this conference.

[6] K. Abe et al., "Electrostatic Design of the Barrel CRID and Associated Measurements, SLAC-PUB--5214, contributed to Vth Intn'l. Conf. on Instrum. for Colliding Beam Physics, Novosibirsk, USSR (1990).

[7] Oxygen Micro Fuel Cell made by Teledyne Analytical Instruments, 16830 Chestnut Street, City of Industry, CA 91748.

[8] M. Cavalli-Sforza, Electron Lifetime Measurements in the Disaster Monitor, CRID Memo 15 (1986).

\title{
DISCLAIMER
}

\begin{abstract}
This report was prepared as an account of work sponsored by an agency of the United States Government. Neither the United States Government nor any agency thereof, nor any of their employees, makes any warranty, express or implied, or assumes any legal liability or responsibility for the accuracy, completeness, or usefulness of any information, apparatus, product, or process disclosed, or represents that its use would not infringe privately owned rights. Reference herein to any specific commercial product, process, or service by trade name, trademark, manufacturer, or otherwise does not necessarily constitute or imply its endorsement, recommendation, or favoring by the United States Government or any agency thereof. The views and opinions of authors expressed herein do not necessarily state or reflect those of the United States Government or any agency thereof.
\end{abstract}



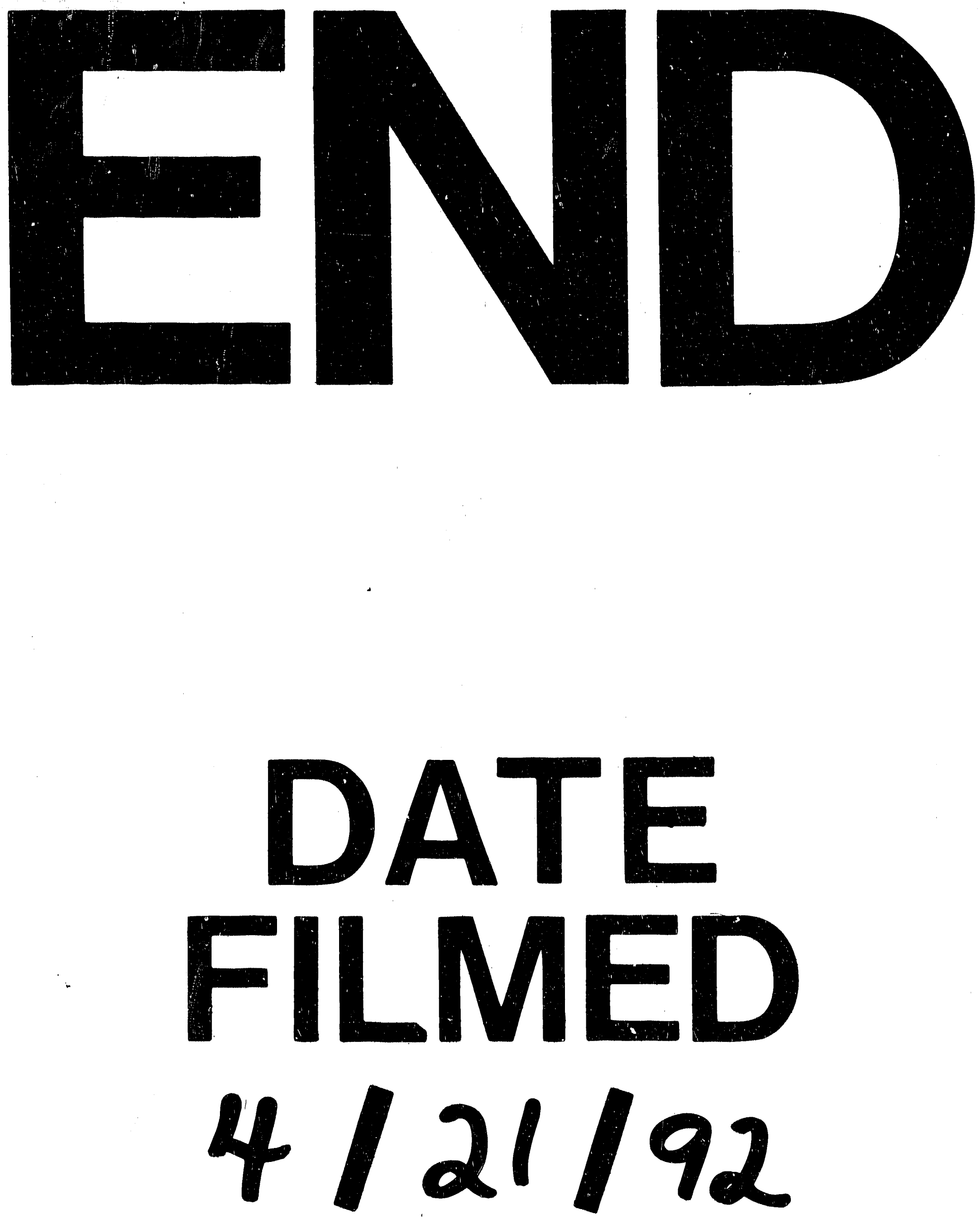

1 
